\title{
The Relationship Between Low-Density Lipoprotein Cholesterol Levels and the Incidence of Cardiovascular Disease in High-Risk Patients Treated With Pravastatin
}

\author{
Main Results of the APPROACH-J Study
}

\author{
Hiroyuki Daida, ${ }^{1}$ MD, Tamio Teramoto, ${ }^{2}$ MD, Yasuhisa Kitagawa, ${ }^{3}$ MD, Yasuyuki Matsushita, ${ }^{4}$ PhD, \\ and Masahiro Sugihara, ${ }^{4} \mathrm{PhD}$, on behalf of the APPROACH-J Study Group
}

\begin{abstract}
SUMMARY
This study aimed to evaluate the relationship between low-density lipoprotein cholesterol (LDL-C) levels and cardiovascular disease (CVD) in high-risk patients with hypercholesterolemia without a history of CVD. Patients who were receiving or started treatment with pravastatin, were followed-up for 2 years. Patients were divided into quartiles according to on-treatment LDL-C. The maximum contrast method based on the Cox proportional hazards model was used to evaluate the relationship between achieved LDL-C and the incidence of CVD. Incidence of CVD was also compared according to whether a number of risk factor targets were achieved. A total 6,229 patients were enrolled, with 4,916 having reported LDL-C values. During the 2 years, 69 cases of CVD (6.7/1000 patient years), including 36 coronary artery disease (CAD) (3.5/1000 patient years) and 28 strokes (2.7/1000 patient years), occurred. The comparison of on-treatment LDL-C level quartiles suggested that the incidence of all CVD decreased linearly as the LDL-C levels decreased. Incidence of CAD showed a curvilinear relationship to LDL-C levels, suggesting some attenuation of risk below LDL-C of $119 \mathrm{mg} / \mathrm{dL}$. The incidence of all CVD and CAD tended to be decreased as the number of achieved risk factor targets increased. In conclusion, through our observational study, it was shown that a linear relationship between the incidence of CVD and LDL-C was observed in high-risk hypercholesterolemic patients. The low incidence of CVD in the present study may be associated with multifactorial management of conventional risk factors including high LDL-C levels. However, prospective, randomized studies are needed to confirm these findings. (Int Heart J 2014; 55: 39-47)
\end{abstract}

Key words: Hypercholesterolemia, Primary prevention, Occlusive atherosclerotic complications, Cardiovascular disease

$\mathrm{D}$ yslipidemia is a consolidated risk factor for atherosclerotic disease, which has been established by a great number of epidemiological studies in Western as well as Asian countries. ${ }^{1-3)}$ The current consensus is that statins can reduce coronary heart disease by $20-40 \%$, with an approximately $30-50 \%$ lowering of low-density lipoprotein cholesterol (LDL-C), irrespective of setting (primary prevention or secondary prevention) ${ }^{4-10)}$ or the presence of other risk factors (eg, diabetes). ${ }^{11)}$

Based on these findings, various guidelines for the diagnosis and treatment of dyslipidemia were conceived and updated to minimize lethal atherosclerotic disease. ${ }^{12,13)}$ In Japan, a treatment guideline for dyslipidemia was first released in 1997, and it has since been modified to become the Japanese Atherosclerosis Society (JAS) Guideline for Diagnosis and Treatment of Hyperlipidemia (2002, updated in 2007). ${ }^{14)}$ The current JAS guideline states that the fundamental treatment concept for pri- mary prevention is modification of lifestyle, including diet and exercise, but if it is difficult to manage lipid levels appropriately, drug therapy including statins should be considered. The JAS guideline also shows the treatment goal for LDL-C level according to stratification by the risk level of a patient. The treatment goals for a patient without conventional risk factors, with one or two risk factors, and with three risk factors or more are set at $<160 \mathrm{mg} / \mathrm{dL},<140 \mathrm{mg} / \mathrm{dL}$, and $<120 \mathrm{mg} / \mathrm{dL}$, respectively, in the primary prevention setting; more intensive goals are recommended for a patient in secondary prevention, based on the findings from clinical trials. ${ }^{15,16)}$ Although the current JAS guideline has been well established based on a large amount of Japanese evidence which has been generated recently, some statements still rely on overseas data. Therefore, we need to accumulate Japanese evidence to confirm whether the recommendation of the JAS guideline is working effectively in the real world setting.

From the ${ }^{1}$ Juntendo University School of Medicine, ${ }^{2}$ Teikyo University School of Medicine, ${ }^{3}$ Tokai University Hachioji Hospital, and ${ }^{4}$ Daiichi Sankyo Co., Ltd., Tokyo, Japan.

This study was conducted by Daiichi Sankyo Co., Ltd. as a post-marketing study according to the good post-marketing study practice guideline. All expenses for conducting this study were provided by Daiichi Sankyo Co., Ltd.

Address for correspondence: Hiroyuki Daida, MD, Department of Cardiology, Juntendo University Graduate School of Medicine, Tokyo, Japan Hongo 3-1-3, Bunkyo-

ku, Tokyo 113-8431, Japan. E-mail: daida@juntendo.ac.jp

Received for publication February 1, 2013

Revised and accepted July 25, 2013.

Released advance online J-STAGE January 27, 2014.

All rights are reserved to the International Heart Journal Association. 
Pravastatin is one of the statins which has firm evidence showing its beneficial effect against cardiovascular disease, ${ }^{17)}$ including studies from Japan. ${ }^{18-21)}$ Of these studies, the landmark MEGA Study clearly revealed that pravastatin significantly reduces cardiovascular disease by $30 \%$, with an $18 \%$ decrease in LDL-C in patients without a history of cardiovascular disease, ${ }^{18)}$ and various findings of the MEGA Study have been cited in the current JAS guideline. ${ }^{15)}$ However, exactly how pravastatin works in high-risk patients in primary prevention categorized by the JAS guideline has not been well investigated to date. Therefore, we investigated the relationship between on-treatment LDL-C level and the incidence of cardiovascular disease in high-risk patients in primary prevention in the real world setting in the Affirmation Primary Prevention with Pravastatin in Reduction of Occlusive Atherosclerotic Complications in Hypercholesterolemia-Japan (APPROACH-J) Study, a prospective observational study of patients taking pravastatin.

\section{MeTHODS}

The design of the APPROACH-J Study has been reported previously. ${ }^{22}$ Briefly, this observational cohort study was started in February 2008 with a target of 5,000 patients to be enrolled until January 2009, and the patients were followed up for 2 years until January 2011.

The patients enrolled in the present study were initiated on or were taking pravastatin at the beginning of the study, and were men aged 20 years or older and women aged 55 years or older (or postmenopausal women) without a history of cardiovascular disease. The patients were categorized as high risk, with 3 or more major risk factors other than high LDL-C level, including being older (age $\geq 45$ years in men, $\geq 55$ years in women), hypertension, diabetes mellitus (including impaired glucose tolerance), smoking, a family history of coronary artery disease, and low high-density lipoprotein cholesterol (HDL-C, $<40 \mathrm{mg} / \mathrm{dL}$ ), according to the JAS guideline. The number of risk factors for each patient was defined based on the physician's diagnosis at the time of enrollment. All enrolled patients were starting or continuing treatment with pravastatin and gave written informed consent. The exclusion criteria were: 1) a history of cardiovascular disease (myocardial infarction, MI; unstable angina; coronary revascularization; or stroke); 2) serious arrhythmia; 3) familial hypercholesterolemia; 4) poorly controlled blood pressure or blood glucose; 5) serious hepatic dysfunction or serious renal dysfunction; 6) other serious diseases, such as malignant tumors; 7) contraindications to pravastatin therapy; and 8) other reasons judged by the investigator to render the patient inappropriate for long-term administration of pravastatin.

Written informed consent was obtained from all eligible patients. Follow-up was continued for 24 months after initiation of the study, irrespective of whether administration of pravastatin continued, and the investigators contacted patients who failed to visit the hospital at 12 and 24 months after initiation of the study to confirm their health status. No restrictions were placed on other treatments, and all treatments were recorded. The patient characteristics, blood pressure laboratory data, compliance with pravastatin therapy, concomitant medications, and presence or absence of cardiovascular events at baseline and after $6,12,18$, and 24 months were recorded in clinical report forms every year. Adverse events were investigated throughout the study period. This study was performed in compliance with the Japanese standards for post-marketing surveillance.

The primary composite endpoint was the first occurrence of cardiovascular disease, which included fatal or nonfatal myocardial infarction, unstable angina requiring hospitalization, coronary revascularization, fatal or nonfatal stroke (except transient ischemic attack), arteriosclerosis obliterans, and sudden and unexpected death. The secondary endpoints were subsets of the primary endpoint, laboratory findings, and safety. All cardiovascular disease reported by the investigators was assessed by the Endpoint Committee.

The study was conducted as a post-marketing study of Daiichi Sankyo Co., Ltd. and fully adhered to the regulations of the Japanese Ministry of Health, Labor and Welfare.

Statistical analysis: The patients who had an on-treatment LDL-C level measured by direct methods were divided into quartiles according to their on-treatment LDL-C levels. All ontreatment LDL-C levels were averaged during follow-up except for those measured before the initiation of pravastatin treatment. Incidence of cardiovascular disease was compared between the quartiles by using the multivariable Cox proportional hazards model, adjusted by sex, age, baseline HDL-C, prior use of antihyperlipidemic agents, family history of coronary artery disease, hypertension, diabetes (including impaired glucose tolerance), body mass index, and smoking status. Furthermore, the maximum contrast method ${ }^{23,24)}$ based on the Cox proportional hazards model was used to investigate the relationships between achieved LDL-C levels and the incidence of cardiovascular events. The maximum contrast method is used to determine the contrast pattern which best fits the observed data with the largest contrast statistic (the smallest $P$-value) ${ }^{24)}$ This method was originally proposed to identify a dose-response pattern, however, it can be also applied to survival data using contrast statistics with several contrast coefficient vectors. ${ }^{25)}$ In this analysis, we evaluated contrast statistics for a regression coefficient vector of the Cox proportional hazards model. The details are described in the Appendix. Additionally, the incidence of cardiovascular disease was compared according to the number of achieved targets for the following factors: LDL-C level, blood pressure, hemoglobin (Hb) A1c, and smoking status. The patients who had mean LDL-C $<120 \mathrm{mg} /$ dL, blood pressure $<130 / 80 \mathrm{mmHg}, \mathrm{HbAlc}$ (US National Glycohemoglobin Standardization Program, NGSP) $<6.5 \%$, and smoking cessation during the follow-up period were defined as having achieved the target for each factor. The mean values during the follow-up period were taken for the achieved values of LDL-C, blood pressure, and HbA1c (NGSP). The blood pressure and HbA1c (NGSP) values measured at each institution by their own methods were used in this study. Smoking status was defined using the baseline data. All statistical analyses were performed using SAS software, version 9.2 (SAS Institute, Cary, NC, USA).

\section{RESUlts}

A total of 6,229 patients were enrolled during a year of 
entry period, and followed up for 2 years. Data from 4,916 patients were used as the final analysis set, after excluding data from 302 patients (including 6 patients who received no pravastatin treatment, 53 patients who did not have any follow-up

Table I. Baseline Characteristics

\begin{tabular}{|c|c|}
\hline & $\begin{array}{l}\text { Final analysis set } \\
\qquad(n=4,916)\end{array}$ \\
\hline Men, $\%$ & 42.2 \\
\hline Age, years & $66.3 \pm 9.9$ \\
\hline $\mathrm{BMI}, \mathrm{kg} / \mathrm{m}^{2}$ & $24.6 \pm 3.9$ \\
\hline Prior hypercholesterolemic medication, $\%$ & 63.6 \\
\hline Prior pravastatin treatment, $\%$ & 58.8 \\
\hline Target LDL-C, ${ }^{*} \mathrm{mg} / \mathrm{dL}$ & 120.8 \\
\hline Receiving dietary instruction, $\%$ & 70.5 \\
\hline Receiving exercise therapy, $\%$ & 59.2 \\
\hline $\mathrm{TC}, \mathrm{mg} / \mathrm{dL}$ & $219.5 \pm 35.1$ \\
\hline $\mathrm{LDL}-\mathrm{C}, \mathrm{mg} / \mathrm{dL}$ & $135.4 \pm 31.1$ \\
\hline HDL-C, mg/dL & $57.5 \pm 15.2$ \\
\hline Non HDL-C, mg/dL & $162.0 \pm 35.5$ \\
\hline Triglycerides, mg/dL (median IQR) & $123.0(90.0-167.0)$ \\
\hline $\mathrm{SBP}, \mathrm{mmHg}$ & $133.8 \pm 16.1$ \\
\hline $\mathrm{DBP}, \mathrm{mmHg}$ & $76.1 \pm 10.9$ \\
\hline Fasting plasma glucose, $\mathrm{mg} / \mathrm{dL}$ & $120.3 \pm 36.1$ \\
\hline HbAlc (NGSP), \% & $6.8 \pm 1.1$ \\
\hline \multicolumn{2}{|l|}{ Conventional risks, $* * \%$} \\
\hline Older age $e^{\S}$ & 95.4 \\
\hline Hypertension & 72.9 \\
\hline Diabetes (including IGT) & 78.1 \\
\hline Smoking & 19.7 \\
\hline Family history of coronary disease & 19.8 \\
\hline Low HDL-C $(<40 \mathrm{mg} / \mathrm{dL})$ & 10.6 \\
\hline Number of risks 3 & 83.7 \\
\hline 4 & 14.5 \\
\hline 5 & 1.7 \\
\hline 6 & 0.2 \\
\hline
\end{tabular}

*Target LDL-C levels were set by patients' physicians. **Conventional risk factors were defined based on physicians' reports. ${ }^{\S} \geq 45$ years for men, $\geq 55$ years for women. BMI indicates body mass index; LDL-C, low-density lipoprotein cholesterol; TC, total cholesterol; HDL-C, highdensity lipoprotein cholesterol; IQR, interquartile range; SBP, systolic blood pressure; DBP, diastolic blood pressure; HbA1c (NGSP), hemoglobin A1c (US National Glycohemoglobin Standardization Program); and IGT, impaired glucose tolerance. data, and 38 patients who withdrew consent), and the 930 patients who did not have on-treatment LDL-C values measured by direct methods. In the follow-up, $95.2 \%$ and $89.3 \%$ of the patients visited the hospital at 12 months and 24 months, respectively. The patients whose data were used in the final analysis (Table I) had a mean age of 66 years; $42.2 \%$ were men and $63.6 \%$ were taking antihyperlipidemic agents, including pravastatin, at enrollment. The baseline LDL-C and HDL-C levels were 219.5 and $57.5 \mathrm{mg} / \mathrm{dL}$. Most of the patients were older $(95.4 \%)$ ( $\geq 45$ years in men, $\geq 55$ years in women) and had hypertension (72.9\%); and/or diabetes, including impaired glucose tolerance (78.1\%). The mean LDL-C during follow-up was $119.5 \mathrm{mg} / \mathrm{dL}$, and there was no apparent change in blood pressure or glucose status (data not shown).

As shown in Table II, a total of 69 cases of cardiovascular disease (6.7/1000 patient years, py), including 36 cases of coronary artery disease (3.5/1000 py), 28 cases of stroke (2.7/1000 py), 3 cases of arteriosclerosis obliterans $(0.3 / 1000$ py), and 2 cases of sudden/cardiac death (0.2/1000 py), occurred during the 2-year follow-up in patients with LDL-C values in the efficacy analysis set. In the 4 groups generated based on the quartiles of on-treatment LDL-C level (Q1, $\leq 104.9 \mathrm{mg} / \mathrm{dL} ; \mathrm{Q} 2$, 105.0 to $<119.0 \mathrm{mg} / \mathrm{dL}$; Q3, 119.0 to $<133.0 \mathrm{mg} / \mathrm{dL}$; and Q4, $\geq 133.0 \mathrm{mg} / \mathrm{dL}$ ), the incidence of all cardiovascular disease decreased as the LDL-C levels decreased. The lowest incidence of all cardiovascular disease was found in group Q1, with the lowest on-treatment LDL-C (hazard ratio, HR, against Q4, $0.431 ; P=0.0210$ ) (Table III).

In testing the suitability for the shapes of L1 to L6 (Figure 1) by the maximum contrast method for all cardiovascular disease, the largest Wald statistic (the smallest $P$-value, 0.0229) was found in the L4 type, suggesting that there is a linear relationship between the incidence of all cardiovascular disease and the LDL-C level (Figure 2A). A curvilinear relationship was found for coronary artery disease and was determined to be of the L2 type, with the largest Wald $\chi^{2}(P=0.0049)$ in the maximum contrast method (Figure $2 \mathrm{~B}$ ). This resulted in the significantly lower incidence of coronary artery disease in group Q2 (HR, 0.348; $P=0.0292$ ) and group Q1 (HR, 0.283; $P=0.0157$ ), shown in Table III. No significant relationship was found between LDL-C and stroke by using the maximum

Table II. Incidences of Events

\begin{tabular}{cc}
\hline Variables & Final analysis set $(n=4,916)$ \\
\hline All cardiovascular diseases & No. of events* (no. of events/1,000 patient years) \\
& $69(6.7)$ \\
Coronary artery disease & $36(3.5)$ \\
Myocardial infarction & $12(1.1)$ \\
Unstable angina & $7(0.7)$ \\
Revascularization & $35(3.4)$ \\
Stroke & $28(2.7)$ \\
Ischemic stroke & $9(0.9)$ \\
Cardioembolic stroke & $1(0.1)$ \\
Lacunar stroke & $9(0.9)$ \\
Hemorrhagic stroke & $9(0.9)$ \\
Subarachnoid hemorrhage & $1(0.1)$ \\
Other strokes & 0 \\
Arteriosclerosis obliterans & $3(0.3)$ \\
Sudden /cardiac death & $2(0.2)$ \\
\hline
\end{tabular}

*First event for each variable. 
Table III. Incidence of Events in Each Quartile Group

\begin{tabular}{lcccc}
\hline \multicolumn{1}{c}{ Group: LDL-C, mean (range), mg/dL } & $\begin{array}{c}\text { No. of events/ } \\
\text { no. of patients }\end{array}$ & $\begin{array}{c}\text { Incidence } \\
(/ 1,000 \mathrm{py})\end{array}$ & $\begin{array}{c}\text { Hazard ratio } \\
(95 \% \mathrm{CI})\end{array}$ & $P$ \\
\hline All cardiovascular diseases & & & & \\
Q1: $92.9(44.0-104.9)$ & $12 / 1,214$ & 4.6 & $0.431(0.211,0.353)$ & 0.0210 \\
Q2: $112.2(105.0-118.8)$ & $17 / 1,240$ & 6.5 & $0.667(0.353,1.258)$ & 0.2106 \\
Q3: $125.5(119.0-132.9)$ & $17 / 1,222$ & 6.6 & $0.732(0.390,1.375)$ & 0.3324 \\
Q4: $147.5(133.0-230.0)$ & $23 / 1,240$ & 8.9 & & - \\
& & & & \\
Coronary artery disease & & & & \\
Q1: $92.9(44.0-104.9)$ & $5 / 1,257$ & 1.9 & $0.283(0.102,0.788)$ & 0.0157 \\
Q2: $112.2(105.0-118.8)$ & $6 / 1,243$ & 2.3 & $0.348(0.135,0.899)$ & 0.0292 \\
Q3: $125.5(119.0-132.9)$ & $9 / 1,224$ & 3.5 & $0.582(0.256,1.320)$ & 0.1948 \\
Q4: $147.5(133.0-230.0)$ & $16 / 1,238$ & 6.2 & & - \\
& & & & 1.00 \\
Stroke & & & & \\
Q1: $93.0(44.0-104.9)$ & $6 / 1,222$ & 2.3 & $0.588(0.191,1.807)$ & 0.3538 \\
Q2: $112.1(105.0-118.7)$ & $9 / 1,230$ & 3.4 & $1.068(0.393,2.903)$ & 0.8977 \\
Q3: $125.5(118.8-132.9)$ & $6 / 1,237$ & 2.3 & $0.769(0.257,2.302)$ & 0.6389 \\
Q4: $147.0(133.0-230.0)$ & $7 / 1,233$ & 2.7 & 1.00 & - \\
\hline
\end{tabular}

LDL-C indicates low-density lipoprotein cholesterol; CI, confidence interval; and py, patient years.
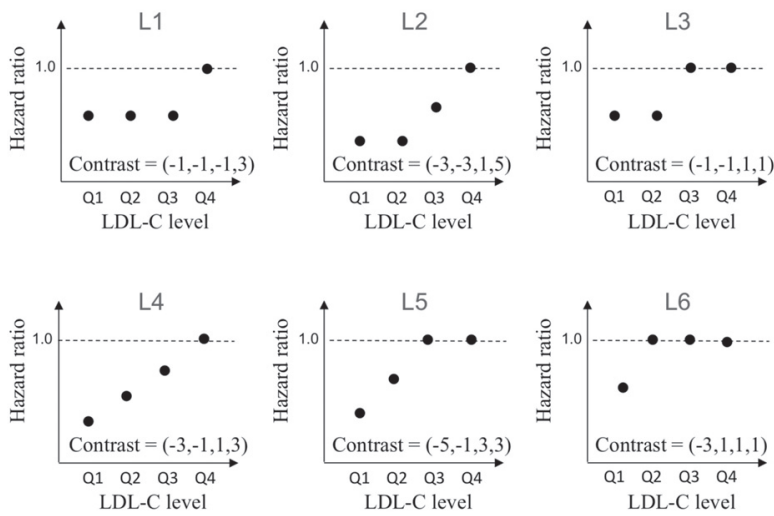

Figure 1. Statistical concepts underlying the maximum contrast method, a statistical method to evaluate the shape of curves which are likely to fit into one of six patterns (L1-L6). LDL-C indicates low-density lipoprotein cholesterol.

contrast method (Figure 2C).

To evaluate the effect of risk factors other than the ontreatment LDL-C level, we compared the baseline features of each quartile. A favorable lipid profile was found in the group with a low on-treatment LDL-C level at baseline. Although the number of patients in each quartile who had hypertension and diabetes mellitus (including impaired glucose tolerance) increased from Q4 to Q1, blood glucose and blood pressure were relatively well controlled within the ranges $118.2-121.7 \mathrm{mg} / \mathrm{dL}$ and 132.9/74.7-134.8/77.7, respectively, between the 4 groups (Table IV). Moreover, antithrombotic agents, in addition to antiplatelet and anticoagulant agents, were used most frequently $(26.4 \%)$ in the group with the lowest LDL-C levels.

As shown in Table V, fewer cases of cardiovascular disease occurred in groups with a greater number of achieved risk factor targets (LDL-C $<120 \mathrm{mg} / \mathrm{dL}$, blood pressure $<130 / 80$ $\mathrm{mmHg}, \mathrm{HbA1c}[\mathrm{NGSP}]<6.5 \%$, and no smoking during follow-up), but this result was not statistically significant $(P=$ $0.239)$. However, the number of cases of coronary artery dis-
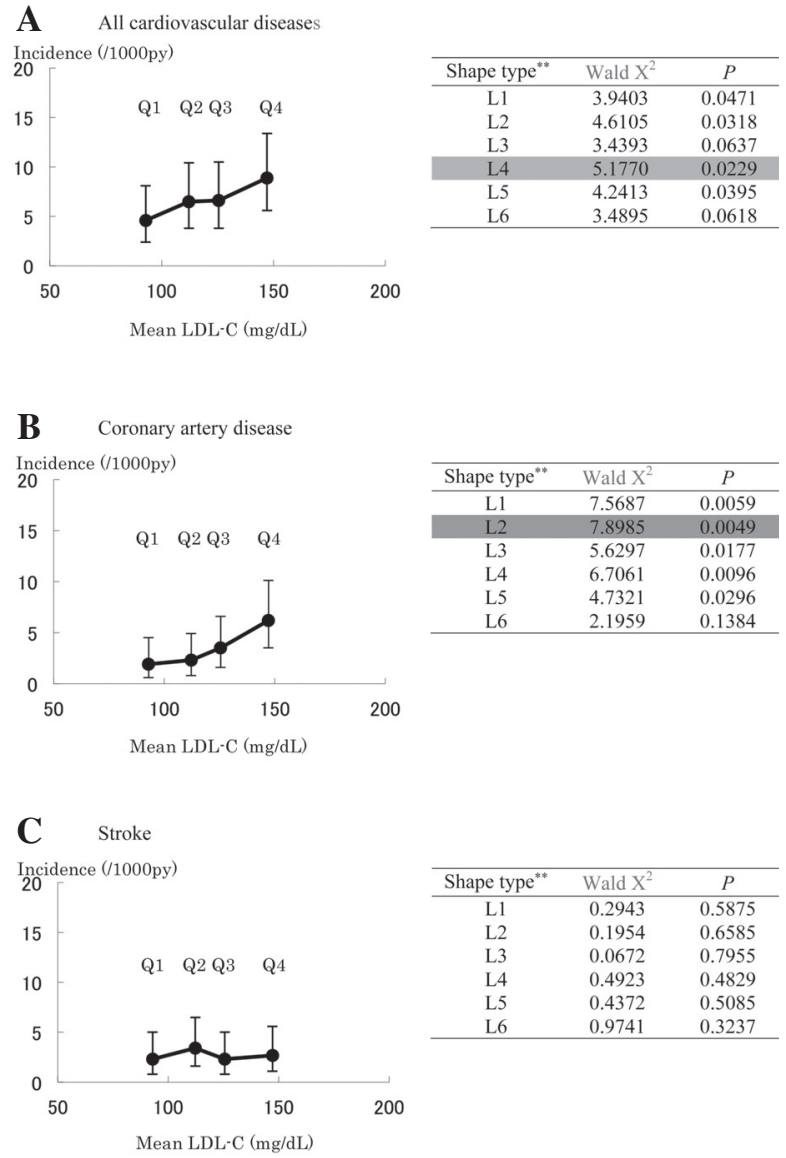

\begin{tabular}{ccc}
\hline Shape type $^{* *}$ & Wald X & $P$ \\
\hline L1 & 0.2943 & 0.5875 \\
L2 & 0.1954 & 0.6585 \\
L3 & 0.0672 & 0.7955 \\
L4 & 0.4923 & 0.4829 \\
L5 & 0.4372 & 0.5085 \\
L6 & 0.9741 & 0.3237
\end{tabular}

Figure 2. Absolute incidence of events and maximum contrast.**The maximum contrast was evaluated by the Cox proportional hazard model, based on logarithmic LDL-C value. **Shape types: refer to Figure 1. LDL-C indicates low-density lipoprotein cholesterol; and py, patient years.

ease was significantly related to the number of risk factor targets achieved $(P=0.021)$; significantly fewer cases of coro- 
Table IV. Baseline Characteristics According to Quartile Group

\begin{tabular}{|c|c|c|c|c|}
\hline Variables & Q1 & Q2 & Q3 & Q4 \\
\hline Men, $\%$ & 46.0 & 40.3 & 39.9 & 42.7 \\
\hline Age, years & 68.7 & 66.9 & 65.9 & 63.8 \\
\hline BMI, $\mathrm{kg} / \mathrm{m}^{2}$ & 24.1 & 24.4 & 24.7 & 25.1 \\
\hline Prior hypercholesterolemic medication & 67.9 & 65.8 & 62.8 & 58.1 \\
\hline Prior pravastatin treatment & 64.4 & 61.3 & 58.5 & 51.0 \\
\hline Target LDL & 117.8 & 120.3 & 121.8 & 123.3 \\
\hline Receiving dietary instruction, \% & 69.5 & 71.9 & 70.6 & 70.0 \\
\hline Receiving exercise instruction, $\%$ & 59.9 & 61.0 & 58.3 & 57.7 \\
\hline $\mathrm{TC}, \mathrm{mg} / \mathrm{dL}$ & 198.2 & 214.4 & 223.6 & 240.0 \\
\hline LDL-C, mg/dL & 113.3 & 128.9 & 140.3 & 157.7 \\
\hline HDL-C, mg/dL & 59.5 & 58.6 & 56.7 & 55.4 \\
\hline Non HDL-C, mg/dL & 139.1 & 156.0 & 167.1 & 180.4 \\
\hline Triglycerides, mg/dL (median) & 109.0 & 113.0 & 124.0 & 138.5 \\
\hline $\mathrm{SBP}, \mathrm{mmHg}$ & 132.9 & 133.5 & 133.9 & 134.8 \\
\hline $\mathrm{DBP}, \mathrm{mmHg}$ & 74.7 & 75.5 & 76.7 & 77.7 \\
\hline Fasting glucose, $\mathrm{mg} / \mathrm{dL}$ & 120.1 & 121.7 & 118.2 & 121.5 \\
\hline HbAlc (NGSP), \% & 6.7 & 6.8 & 6.8 & 6.9 \\
\hline \multicolumn{5}{|l|}{ Conventional risk factor, $* * \%$} \\
\hline Age $\S$ & 97.4 & 96.8 & 95.3 & 92.1 \\
\hline Hypertension & 76.5 & 72.5 & 71.7 & 71.1 \\
\hline Diabetes (including IGT) & 83.0 & 79.2 & 75.1 & 74.9 \\
\hline Smoking & 19.1 & 18.3 & 19.3 & 22.1 \\
\hline Family history of coronary disease & 15.2 & 18.1 & 22.1 & 23.6 \\
\hline Low HDL-C $(<40 \mathrm{mg} / \mathrm{dL})$ & 9.2 & 10.5 & 10.9 & 11.7 \\
\hline \multicolumn{5}{|l|}{ Medication, \% } \\
\hline Antihypercholesterolemic agents & 10.9 & 11.3 & 11.4 & 14.0 \\
\hline Statins & 5.5 & 6.6 & 5.8 & 8.5 \\
\hline Simvastatin & 0.1 & 0 & 0.2 & 0.1 \\
\hline Fluvastatin & 0 & 0 & 0.1 & 0.4 \\
\hline Atorvastatin & 1.6 & 2.2 & 0.9 & 1.5 \\
\hline Pitavastatin & 1.4 & 1.1 & 1.8 & 2.8 \\
\hline Rosuvastatin & 2.8 & 3.5 & 3.2 & 4.0 \\
\hline Fibrates & 1.3 & 1.2 & 1.1 & 1.0 \\
\hline Others & 4.7 & 4.4 & 4.9 & 5.5 \\
\hline Antihypertensive agents & 74.3 & 69.8 & 67.4 & 63.6 \\
\hline Antidiabetic agents & 60.0 & 56.7 & 48.9 & 47.4 \\
\hline Antithrombotic agents & 26.4 & 18.1 & 16.9 & 14.3 \\
\hline
\end{tabular}

**Conventional risk factors were defined based on physicians' reports. ${ }^{\S} \geq 45$ years for men, $\geq 55$ years for women. BMI indicates body mass index; LDL-C, low-density lipoprotein cholesterol; TC, total cholesterol; HDL-C, high-density lipoprotein cholesterol; SBP, systolic blood pressure; DBP, diastolic blood pressure; HbA1c (NGSP), hemoglobin A1c (US National Glycohemoglobin Standardization Program); and IGT, impaired glucose tolerance.

nary artery disease were found in patients who achieved targets for 3 risk factors (HR, 0.216, $P=0.031$ ). No relationship was found between the number of risk factor targets achieved and the incidence of stroke $(P=0.561)$.

In the 6,053 patients who had pravastatin at least once during the 2-year follow-up, $320(5.3 \%)$ experienced severe adverse events, including cancer (95 patients, $1.6 \%$ ), pneumonia ( 9 patients, $0.1 \%$ ), and atrial fibrillation ( 8 patients, $0.1 \%$ ). Frequent reports of nonsevere adverse events included increased creatine phosphokinase (62 patients, $1.0 \%$ ) and muscle ache (38 patients, $0.6 \%$ ). There were also 175 adverse drug reactions, 6 of which were serious (thrombocytopenia, adult-onset Still's disease, breast cancer, VIIth nerve paralysis, urinary calculus, and hospitalization), which were judged by physicians as being possibly related to pravastatin.

\section{Discussion}

There is a paucity of information in Japan on the evaluation of the relationship between LDL-C and cardiovascular disease in high-risk patients in the primary prevention setting, such as those with 3 or more cardiovascular risk factors other than high LDL-C. For these high-risk patients, the JAS guideline recommends the reduction of LDL-C level to $<120 \mathrm{mg}$ / $\mathrm{dL}$ by improving their lifestyle. However, a large number of patients allocated to this category receive a statin to achieve their target LDL-C in general practice. Therefore, it is still important to investigate the relationship between on-treatment LDL-C level after taking statin treatment and the onset of cardiovascular disease.

The APPROACH-J Study provides some important findings. First, the incidence of cardiovascular disease was low even though $>70 \%$ of the patients had diabetes, hypertension, 
Table V. Relationship Between Incidence of Vascular Disease and Number of Risk Factor Targets Achieved

\begin{tabular}{|c|c|c|c|c|c|c|c|c|c|}
\hline \multirow[b]{2}{*}{$\begin{array}{c}\text { No. of } \\
\text { targets } \\
\text { achieved* }\end{array}$} & \multicolumn{3}{|c|}{ All cardiovascular diseases } & \multicolumn{3}{|c|}{ Coronary artery disease } & \multicolumn{3}{|c|}{ Stroke** } \\
\hline & $\begin{array}{l}\text { No. of events/ } \\
\text { no. of patients } \\
\text { (no. of } \\
\text { events } 1,000 \\
\text { py) }\end{array}$ & $\begin{array}{l}\text { Hazard ratio } \\
(95 \% \mathrm{CI})\end{array}$ & $P$ & $\begin{array}{l}\text { No. of events/ } \\
\text { no. of patients } \\
\text { (no. of } \\
\text { events/1,000 } \\
\text { py) }\end{array}$ & $\begin{array}{l}\text { Hazard ratio } \\
(95 \% \mathrm{CI})\end{array}$ & $P$ & $\begin{array}{l}\text { No. of events/ } \\
\text { no. of patients } \\
\text { (no. of } \\
\text { events/1,000 } \\
\text { py) }\end{array}$ & $\begin{array}{l}\text { Hazard ratio } \\
(95 \% \mathrm{CI})\end{array}$ & $P$ \\
\hline 0 & $\begin{array}{l}3 / 125 \\
(11.3)\end{array}$ & - & $0.239^{\S}$ & $\begin{array}{l}3 / 125 \\
(11.3)\end{array}$ & - & $0.021^{\S}$ & $\begin{array}{c}0 / 124 \\
(0.0)\end{array}$ & & \\
\hline 1 & $\begin{array}{c}15 / 867 \\
(8.2)\end{array}$ & $\begin{array}{c}0.725 \\
(0.210-2.504)\end{array}$ & 0.611 & $\begin{array}{c}10 / 868 \\
(5.4)\end{array}$ & $\begin{array}{c}0.482 \\
(0.133-1.751)\end{array}$ & 0.267 & $\begin{array}{l}4 / 868 \\
(2.2)\end{array}$ & - & $0.561^{8}$ \\
\hline 2 & $\begin{array}{c}23 / 1,573 \\
(6.9)\end{array}$ & $\begin{array}{c}0.611 \\
(0.184-2.037)\end{array}$ & 0.423 & $\begin{array}{c}12 / 1,573 \\
(3.6)\end{array}$ & $\begin{array}{c}0.318 \\
(0.090-1.126)\end{array}$ & 0.076 & $\begin{array}{c}10 / 1,574 \\
(3.0)\end{array}$ & $\begin{array}{c}1.383 \\
(0.434-4.408)\end{array}$ & 0.584 \\
\hline 3 & $\begin{array}{c}14 / 1,148 \\
(5.7)\end{array}$ & $\begin{array}{c}0.505 \\
(0.145-1.758)\end{array}$ & 0.283 & $\begin{array}{c}6 / 1,150 \\
(2.5)\end{array}$ & $\begin{array}{c}0.216 \\
(0.054-0.866)\end{array}$ & 0.031 & $\begin{array}{c}6 / 1,151 \\
(2.4)\end{array}$ & $\begin{array}{c}1.122 \\
(0.317-3.976)\end{array}$ & 0.859 \\
\hline 4 & $\begin{array}{c}2 / 302 \\
(3.1)\end{array}$ & $\begin{array}{c}0.271 \\
(0.045-1.624)\end{array}$ & 0.153 & $\begin{array}{c}0 / 303 \\
(0.0)\end{array}$ & - & - & $\begin{array}{c}2 / 302 \\
(3.1)\end{array}$ & $\begin{array}{c}1.413 \\
(0.259-7.717)\end{array}$ & 0.690 \\
\hline
\end{tabular}

*Achievement of risk factor targets was defined by using mean values during follow-up according to the following criteria: low-density lipoprotein cholesterol < $120 \mathrm{mg} / \mathrm{dL}$, blood pressure $<130 / 80 \mathrm{mmHg}$, hemoglobin Alc (US National Glycohemoglobin Standardization Program) < 6.5\%, and no smoking during follow-up. **The hazard ratio was calculated in relation to the combination group consisting of groups 0 and 1 , because there were no events in group 0 . py indicates, patient years; and CI, confidence interval. ${ }^{8}$ The $P$ value is for the number of risk factor targets achieved, included as a quantitative variable in the multivariable Cox proportional hazards model.

or both. In the present study, only 69 cardiovascular events occurred in 4,916 patients during the 2 years (6.7/1000 py), corresponding to approximately $0.7 \%$ per year. This incidence is one-third that of estimates derived from data used in the subanalysis of the MEGA Study for diabetes, ${ }^{26)}$ whose participants had similar background characteristics to those of patients enrolled in the present study. Although it is necessary to take into account major differences in dealing with angina between the two studies (only unstable angina was included as the primary endpoint in APPROACH-J; in contrast, all angina events, including stable ones, were included in the MEGA Study), the incidence of MI and stroke was still low in APPROACH-J compared with in the MEGA diabetic population (1.1 versus $1.9 / 1000$ py for MI and 2.7 versus $4.1 / 1000$ py for stroke). The difference in the incidence of cardiovascular disease between the two studies may suggest that there are some differences between the clinical trial and general practice settings. In fact, other epidemiological data from Japan similarly showed a low incidence of cardiovascular disease even though the studies involved high-risk patients. ${ }^{27-29)}$ It should also be considered that recent clinical practice tends to involve treating patients more aggressively, through the popularization of several guidelines, which might have reduced event rates in the Japanese population.

The second point is that the results of this study clearly show that the achievement of low levels of LDL-C is highly associated with a low incidence of cardiovascular disease in these patients. Even though other conventional risk factors were well-controlled, the influence of LDL-C clearly remains. This finding suggests that an aggressive LDL-C-lowering strategy could be applied to high-risk patients, although the absolute event rate appears to be low even in this group.

When looking at coronary events and cerebral events separately, a significant relationship with LDL-C level was found for coronary events, reaching some attenuation of risk and a plateau in Q2 and Q1, in which the LDL-C level was < 119 $\mathrm{mg} / \mathrm{dL}$, but not for cerebral events. The relationships between LDL-C and coronary events and stroke observed in this study are similar to those reported for coronary events ${ }^{27,30-32)}$ and cerebral events. ${ }^{33-35)}$ The Japan Lipid Intervention Trial (J-LIT), an observational study with simvastatin, similarly showed that the incidence of coronary artery disease reaches a plateau level at LDL-C around $120-140 \mathrm{mg} / \mathrm{dL}$ in a primary prevention setting. ${ }^{26)}$ Also, the post-hoc analysis of the MEGA Study, which investigated the relationship between on-treatment LDL-C level and coronary heart disease and stroke, showed a similar curvilinear shape in relation to on-treatment LDL-C levels and coronary artery disease and stroke. The results of these studies also showed that the curve levels out around LDL-C $120 \mathrm{mg}$ / $\mathrm{dL}$ in coronary artery disease, and they showed no relationship between LDL-C levels and stroke, consistent with the results of the present study. ${ }^{32)}$ Therefore, these findings are consistent with the LDL-C target for primary prevention recommended by the 2007 JAS guideline. ${ }^{14)}$ However, the efficacy of LDL-C lowering for stroke prevention and target levels of LDL-C for coronary artery disease prevention in the Japanese population, especially in high-risk patients, should be further investigated.

Generally, risk for cardiovascular disease is not only related to LDL-C levels but also to blood pressure, hyperglycemia, and smoking. Recent research has revealed that a greater number of cardiovascular health metrics are associated with a lower risk of cardiovascular disease mortality, the 7 cardiovascular health metrics being not smoking, being physically active, having normal blood pressure, having normal blood glucose, having low total cholesterol, having an ideal weight, and eating a healthy diet. ${ }^{36)}$ The present study revealed that blood pressure and $\mathrm{HbA} 1 \mathrm{c}$ value decrease as LDL-C levels decrease. Therefore, we attempted to determine the relationship between cardiovascular disease and control of these 4 factors (blood pressure, HbA1c, smoking, and LDL-C level). The results showed that the incidence of cardiovascular disease decreases as more risk factor targets are achieved. (The targets are defined by the guidelines of the JAS, ${ }^{14)}$ Japan Society of Hypertension, ${ }^{37)}$ and Japan Diabetes Society ${ }^{38)}$ ). These results indicate that patients who achieve low LDL-C and concurrently have good blood pressure and glucose control, as well as good 
management of conventional factors, may have the associated low incidence of cardiovascular disease in the lowest LDL-C group. Furthermore, the use of antithrombotic agents, which has widely been recognized for providing a beneficial effect in the prevention of cardiovascular disease, was greater in the lower LDL-C group (Table IV). Moreover, we have evaluated adherence to lipid-lowering treatment as another objective in this study, and we found that good adherence to drug therapy is associated with lower LDL-C level. ${ }^{39)}$ In this study, about $90 \%$ of the patients had good adherence to drug therapy including pravastatin. These two facts may explain the low incidence of cardiovascular disease.

The present study had some limitations related to its design. The population of this study was not selected randomly. Patients invited to participate in the study were considered to be in sufficiently good condition to tolerate the long-term follow-up. This may result in an event rate lower than that in the actual population who are at high risk in primary prevention. In addition, the inclusion criteria of the present study allowed patients to be enrolled who were continuing pravastatin at initiation of the study, resulting in a mixture of pravastatin-naïve and non-naïve patients. In fact, about half of the patients had taken pravastatin at enrollment. However, we believe that these facts do not affect the interpretation of the study results, because there were no apparent differences in baseline characteristics or in the incidence of events between pravastatin-naïve and non-naive patients (data not shown). This belief is supported by the fact that there was little impact with adjustment for pre-pravastatin treatment (HR for group with pre-hypercholesterolemic drugs against those without them, $0.94 ; P=0.83$ in the multivariable Cox proportional hazards model).

The LDL-C values that we used in this analysis were all obtained by direct methods, and there was no distinction as to the types of kit which were included in the analysis. Recently, it was reported that the Friedewald method is more appropriate for determining LDL-C values than direct methods, because of the great variability between different measuring kits. ${ }^{40)}$ However, we decided to adopt direct methods in this analysis because of the lack of a great number of total cholesterol values, which is related to the reimbursement policy in some regions. Many institutions were not allowed to measure both total cholesterol and LDL-C by direct methods during the study period. Moreover, blood samples were not taken while patients were fasting, which also had an effect on the lack of Friedewald LDL-C values. Only $33.5 \%$ of samples provided enough data for the Friedewald method in this study setting. However, although we only used LDL-C values obtained by direct methods, the results of the study could apply irrespective of measurement methods, because the correlation between the values obtained by direct methods and those obtained by Friedewald's methods was high $(r=0.90)$ in the analysis of 3,769 LDL-C values in 1,681 patients who had both LDL-C and Friedewald's values.

The follow-up period was rather short; 2 years may not reflect the actual treatment period in general practice. Moreover, we did not obtain information about the duration of patients' high LDL-C condition, and how long they had been receiving lipid-lowering treatment before enrollment; these data would be different for individual patients. A long-term state of high LDL-C before enrollment may affect the incidence of cardiovascular disease, and it should be taken into account when interpreting the findings of the present study, especially in this kind of short-term observation. The multiplicity adjustment is generally essential for confirmatory studies conducted with the purpose of proving a certain hypothesis from among many statistical tests in order to maintain the total alpha error within the statistical significance level. Since this study is an observational study mainly to identify the relationships between achieved LDL-C and the incidence of cardiovascular events, there was no multiplicity adjustment in the analysis. Finally, since this study was an observational study, we did not consult the physicians and patients about not only lipid levels but also other risk factors. In the analysis for the number of risk factors for which target levels were achieved, the achievement of targets was defined under natural conditions in the clinical setting, meaning that a naturally low-risk population may be included in the achievement group who might have had different characteristics compared with treated patients.

In conclusion, through our observational study design, it was shown that high-risk patients in the primary prevention setting receiving pravastatin treatment have a low incidence of cardiovascular disease, which may be associated with good control of conventional risk factors. It also shows that a lower LDL-C level is associated with a lower incidence of cardiovascular disease. Pravastatin is used broadly in the primary prevention setting based on consolidated evidence, ${ }^{18)}$ and the findings from the present study show that it should still be considered for high-risk hypercholesterolemic patients in a primary prevention setting, along with managing blood pressure and $\mathrm{HbA} 1 \mathrm{c}$ according to the guidelines, and abstaining from smoking. The updated JAS guideline for 2012 has been released, ${ }^{41)}$ and it emphasizes the importance of managing multiple risk factors, including hypertension, diabetes, and smoking, along with lipid management, as a total risk management concept. The findings of the present study support the idea that total risk management could be associated with a low incidence of cardiovascular disease. However, prospective, randomized studies are needed to confirm these findings.

\section{ACKNOWLEDGMENTS}

We are very grateful to all investigators for their cooperation in the APPROACH-J Study.

\section{APPENDIX}

Maximum contrast method based on the Cox proportional hazards model

The Cox proportional hazards model with a regression parameter vector $\beta$ is expressed as follows:

$$
\left.\lambda(t)=\lambda_{0}(t) \exp \left(\beta^{\prime} Z\right)\right)
$$

where $\lambda(t)$ is the hazard for an event at time $t, \lambda_{0}(t)$ is an arbitrary and unspecified baseline hazard function, and $\mathrm{Z}$ is a vector of explanatory variables (quartiles of achieved LDL-C). The regression parameter vector $\beta$ is estimated using a partial likelihood method.

In order to detect a response pattern which best fits observed data among candidate patterns, contrast coefficient vectors corresponding to the candidate patterns are specified. Let $l_{i}$ be the ith contrast coefficient vector to test the null hypothesis $\mathrm{H}_{0}: \mathrm{l}_{i}^{\prime} \beta=0$. Then, the covariance matrix of a contrast function $\mathrm{l}_{i}^{\prime} \beta$ is given by $\mathrm{l}_{i}^{\prime} \mathrm{V}(\beta) \mathrm{l}_{i}$, where $\mathrm{V}(\beta)$ is a model- 
based covariance matrix of $\beta$. The contrast statistic for the contrast coefficient vector $1_{i}$ is formalized as follows:

$$
\chi^{2}=\left(\mathrm{l}_{i}^{\prime} \beta\right)^{\prime}\left(\mathrm{l}_{i}^{\prime} \mathrm{V}(\beta) \mathrm{l}_{i}\right)^{-1} \mathrm{l}_{i} \beta .
$$

The maximum contrast method identifies a response pattern as the corresponding one which achieves the maximum of the contrast statistics. ${ }^{24)}$ The contrast statistic is approximated by a chi-square distribution with 1 degree of freedom where $\left(\mathrm{l}_{i}^{\prime} \mathrm{V}(\beta) \mathrm{l}_{i}\right)^{-1}$ is an inverse matrix of $\mathrm{l}_{i}^{\prime} \mathrm{V}(\beta) \mathrm{l}_{i}$ for large samples under the null hypothesis $\mathrm{H}_{0}{ }^{42)}$

A list of the participating physicians is available on the website. (https://www.jstage.jst.go.jp/article/ihj/55/1/55_13-002/_article)

\section{REFERENCES}

1. Asia Pacific Cohort Studies Collaboration. Joint effects of systolic blood pressure and serum cholesterol on cardiovascular disease in the Asia Pacific region. Circulation 2005; 112: 3384-90.

2. Kannel WB, Castelli WP, Gordon T, McNamara PM. Serum cholesterol, lipoproteins, and the risk of coronary heart disease. The Framingham study. Ann Intern Med 1971; 74: 1-12.

3. Wilhelmsen L. ESC Population Studies Lecture 1996. Cardiovascular monitoring of a city over 30 years. Eur Heart J 1997; 18: 1220-30.

4. Scandinavian Simvas Survival Study Group. Randomised trial of cholesterol lowering in 4444 patients with coronary heart disease: the Scandinavian Simvastatin Survival Study (4S). Lancet 1994; 344: 1383-9.

5. Shepherd J, Cobbe SM, Ford I, et al. Prevention of coronary heart disease with pravastatin in men with hypercholesterolemia. West of Scotland Coronary Prevention Study Group. N Engl J Med 1995; 333: 1301-7.

6. Downs JR, Clearfield M, Weis S, et al. Primary prevention of acute coronary events with lovastatin in men and women with average cholesterol levels: results of AFCAPS/TexCAPS. Air Force/ Texas Coronary Atherosclerosis Prevention Study. JAMA 1998; 279: 1615-22.

7. Ridker PM, Danielson E, Fonseca FA, et al. Rosuvastatin to prevent cardiovascular events in men and women with elevated C-reactive protein. N Engl J Med 2008; 359: 2195-207.

8. Schwartz GG, Olsson AG, Ezekowitz MD, et al. Effects of atorvastatin on early recurrent ischemic events in acute coronary syndromes: the MIRACL study: a randomized controlled trial. JAMA 2001; 285: 1711-8

9. LaRosa JC, Grundy SM, Waters DD, et al. Intensive lipid lowering with atorvastatin in patients with stable coronary artery disease. N Engl J Med 2005; 352: 1425-35.

10. Action to Control Cardiovascular Risk in Diabetes Study Group, Gerstein HC, Miller ME, Byington RP, et al. Effects of intensive glucose lowering in type 2 diabetes. N Engl J Med 2008; 358: 2545-59.

11. Colhoun HM, Betteridge DJ, Durrington PN, et al. Primary prevention of cardiovascular disease with atorvastatin in type 2 diabetes in the Collaborative Atorvastatin Diabetes Study (CARDS): multicentre randomised placebo-controlled trial. Lancet 2004; 364: 685-96.

12. Expert Panel on Detection, Evaluation, and Treatment of High Blood Cholesterol in Adults. Executive Summary of The Third Report of The National Cholesterol Education Program (NCEP) Expert Panel on Detection, Evaluation, and Treatment of High Blood Cholesterol in Adults (Adult Treatment Panel III). JAMA 2001; 285: 2486-97.

13. Task Force for the management of dyslipidaemias of the European Society of Cardiology (ESC) and the European Atherosclerosis Society (EAS), Catapano AL, Reiner Z, De Backer, et al. ESC/ EAS Guidelines for the management of dyslipidaemias: the Task Force for the management of dyslipidaemias of the European Society of Cardiology (ESC) and the European Atherosclerosis Soci- ety (EAS). Atherosclerosis 2011; 217: S1-44.

14. Hata Y, Mabuchi H, Saito Y, et al. Report of the Japan Atherosclerosis Society (JAS) Guideline for Diagnosis and Treatment of Hyperlipidemia in Japanese adults. J Atheroscler Thromb 2002; 9: $1-27$.

15. Teramoto T, Sasaki J, Ueshima H, et al. Executive summary of Japan Atherosclerosis Society (JAS) guideline for diagnosis and prevention of atherosclerotic cardiocardiovascular diseases for Japanese. J Atheroscler Thromb 2007; 14: 45-50. (Review)

16. Smith SC Jr, Allen J, Blair SN, et al. AHA/ACC guidelines for secondary prevention for patients with coronary and other atherosclerotic vascular disease: 2006 update: endorsed by the National Heart, Lung, and Blood Institute. Circulation 2006; 113: 2363-72.

17. Haria M, McTavish D. Pravastatin. A reappraisal of its pharmacological properties and clinical effectiveness in the management of coronary heart disease. Drugs 1997; 53: 299-336. (Review)

18. Nakamura $\mathrm{H}$, Arakawa $\mathrm{K}$, Itakura $\mathrm{H}$, et al. Primary prevention of cardiovascular disease with pravastatin in Japan (MEGA Study): a prospective randomised controlled trial. Lancet 2006; 368: 115563.

19. Ito H, Ouchi Y, Ohashi Y, et al. A comparison of low versus standard dose pravastatin therapy for the prevention of cardiovascular events in the elderly: the pravastatin anti-atherosclerosis trial in the elderly (PATE). J Atheroscler Thromb 2001; 8: 33-44.

20. The Kyushu Lipid Intervention Study Group. Pravastatin use and risk of coronary events and cerebral infarction in Japanese men with moderate hypercholesterolemia: the Kyushu Lipid Intervention Study. J Atheroscler Thromb 2000; 7: 110-21.

21. Saeki T, Sakuma N, Hayakawa K, et al. Low incidence of cardiac events in statin-administered patients in CAG study. J Atheroscler Thromb 2009; 16: 172-8.

22. Teramoto T, Kitagawa Y, Daida H; APPROACH-J Study group. APPROACH-J study: design, rationale, and baseline data of the affirmation primary prevention with pravastatin in reduction of occlusive atherosclerotic complications in hypercholesterolemia--Japan study. J Atheroscler Thromb 2011; 18: 1054-61.

23. Wakana A, Yoshimura I, Hamada C. A method for therapeutic dose selection in a phase II clinical trial using contrast statistics. Stat Med 2007; 26: 498-511.

24. Yoshimura I, Wakana A, Hamada C. A performance comparison of maximum contrast methods to detect dose dependency. Drug Inf J 1997; 31: 423-32.

25. Sugihara M. Survival analysis using inverse probability of treatment weighted methods based on the generalized propensity score. Pharm Stat 2010; 9: 21-34

26. Tajima N, Kurata $\mathrm{H}$, Nakaya N, et al. Pravastatin reduces the risk for cardiovascular disease in Japanese hypercholesterolemic patients with impaired fasting glucose or diabetes: diabetes subanalysis of the Management of Elevated Cholesterol in the Primary Prevention Group of Adult Japanese (MEGA) Study. Atherosclerosis 2008; 199: 455-62.

27. Matsuzaki M, Kita T, Mabuchi H, et al; Japan Lipid Intervention Trial. Large scale cohort study of the relationship between serum cholesterol concentration and coronary events with low-dose simvastatin therapy in Japanese patients with hypercholesterolemia. Circ J 2002; 66: 1087-95.

28. Kawamori R, Fujita T, Matsuoka H, Umemura S, Saito Y. Relation between cardiovascular complications and blood pressure/blood glucose control in diabetic patients with hypertension receiving long-term candesartan cilexetil therapy: Challenge-DM study. Diabetes Res Clin Pract 2009; 83: 241-8.

29. Tanabe N, Iso H, Okada K, et al. Serum total and non-high-density lipoprotein cholesterol and the risk prediction of cardiovascular events - the JALS-ECC -. Circ J 2010; 74: 1346-56.

30. West of Scotland Coronary Prevention Study Group. Influence of pravastatin and plasma lipids on clinical events in the West of Scotland Coronary Prevention Study (WOSCOPS). Circulation 1998; 97: 1440-5.

31. Simes RJ, Marschner IC, Hunt D, et al. Relationship between lipid levels and clinical outcomes in the Long-term Intervention with 
Pravastatin in Ischemic Disease (LIPID) Trial: to what extent is the reduction in coronary events with pravastatin explained by onstudy lipid levels? Circulation 2002; 105: 1162-9.

32. Teramoto T, Nakaya N, Yokoyama S, Ohashi Y, Mizuno K, Nakamura H. Association between lowering low-density lipoprotein cholesterol with pravastatin and primary prevention of cardiovascular disease in mild to moderate hypercholesterolemic Japanese. J Atheroscler Thromb 2010; 17: 879-87.

33. Prospective Studies Collaboration. Cholesterol, diastolic blood pressure, and stroke: 13,000 strokes in 450,000 people in 45 prospective cohorts. Lancet 1995; 346: 1647-53.

34. Imamura T, Doi Y, Arima H, et al. LDL cholesterol and the development of stroke subtypes and coronary heart disease in a general Japanese population: the Hisayama study. Stroke 2009; 40: 382-8.

35. Uchiyama S, Nakaya N, Mizuno K, et al. Risk factors for stroke and lipid-lowering effect of pravastatin on the risk of stroke in Japanese patients with hypercholesterolemia: analysis of data from the MEGA Study, a large randomized controlled trial. J Neurol Sci 2009; 284: 72-6.

36. Yang Q, Cogswell ME, Flanders WD, et al. Trends in cardiovascular health metrics and associations with all-cause and CVD mortality among US adults. JAMA 2012; 307: 1273-83.
37. Ogihara T, Kikuchi K, Matsuoka H, et al. The Japanese Society of Hypertension Guidelines for the Management of Hypertension (JSH 2009). Hypertens Res 2009; 32: 3-107.

38. Neville SE, Boye KS, Montgomery WS, Iwamoto K, Okamura M, Hayes RP. Diabetes in Japan: a review of disease burden and approaches to treatment. Diabetes Metab Res Rev 2009; 25: 705-16. (Review)

39. Kitagawa Y, Teramoto T, Daida H. Adherence to preferable behavior for lipid control by high-risk dyslipidemic Japanese patients under pravastatin treatment: the APPROACH-J study. J Atheroscler Thromb 2012; 19: 795-805.

40. Miller WG, Myers GL, Sakurabayashi I, et al. Seven direct methods for measuring HDL and LDL cholesterol compared with ultracentrifugation reference measurement procedures. Clin Chem 2010; 56: 977-86.

41. Japan Atherosclerosis Society eds. Japan Atherosclerosis Society (JAS) Guidelines for Prevention of Atherosclerotic Cardiovascular Diseases 2012. Tokyo, Japan: Japan Atherosclerosis Society; 2012.

42. Peto R, Peto J. Asymptotically efficient rank invariant test procedures. J R Stat Soc Ser A 1972; 135: 185-207. 